\section{Ein Krankenhaus ist keine Rückenschule}

Immer mehr Patienten haben „Rücken“ und liegen damit im Krankenhaus, ohne dass ihnen geholfen wird. Und das, obwohl immer öfter operiert wird.

$\mathrm{D}$ iese Meldung, von den Krankenkassen in Umlauf gebracht, wurde auf der Titelseite der BILD-Zeitung quasi als Sensation verkauft. Dabei dürfte allseits bekannt sein, dass ein Krankenhaus keine Rückenschule ist. Im Gegenteil, alle, die schon einmal im Krankenhaus gelegen haben, wissen um die Qualität der Matratzen dort. Wenn man nicht im Krankenhaus Rückenschmerzen bekommt, ja wo denn dann? Viele Patienten, die primär wegen einer anderen Erkrankung stationär behandelt werden, gehen bereits nach einigen Tagen mit "Rücken“ nach Hause. Böse Zungen behaupten sogar, die Matratzen seien ein willkommenes Mittel, um die Klinikverweildauer zu verkürzen. Allzu oft wird versucht, dem Massenphänomen „Rü- ckenschmerzen" mit Röntgen, CT und MRT entgegenzuwirken, leider ohne nennenswerten Erfolg. Kaum ein Patient verspürt nämlich Besserung, wenn er die Segnungen einer überzogenen Diagnostik, die zwar nicht Evidenz-, aber Eminenz-basiert ist, genießen durfte. Doch wenn Rückenschmerzen im Krankenhaus eher schlimmer als besser werden, sollte man Patienten dringend davon abraten, ein solches wegen solcher Beschwerden überhaupt aufzusuchen. Gleiches gilt für den gar nicht so seltenen Fall, dass der Rücken unter's Messer soll. „Rücken“ braucht eben Geduld und Zeit, bis die Natur sie geheilt hat. Und die Natur lässt sich bisweilen gerade bei „Rücken“ viel, viel Zeit.

(Dr. P. Stiefelhagen)

\title{
Arzneimitteltherapie muss sicherer werden
}

\section{Viele Pflegeheimbewohner leiden an einer eingeschränkten Nierenfunktion und nehmen gleichzeitig dauerhaft zahlreiche Medikamente ein. Ein nicht zu unterschätzendes Gesundheitsrisiko, wie die Studie "Heim trifft Niere" belegt.}

\begin{abstract}
m Rahmen des Forschungsprojektes I,Inappropriate Medication in Patients with Renal insufficiency in Nursing Homes" (IMREN) untersuchten Versorgungsforscher der Universitäten Oldenburg und Bremen erstmalig für Deutschland, wie viele Pflegeheimbewohner eine Niereninsuffizienz haben und wie häufig sie Medikamente nehmen, die dafür nicht angemessen dosiert sind. Dazu wurden Informationen von 852 Bewohnern aus Heimen in Bremen und Niedersachsen ausgewertet.
\end{abstract}

Die Zahlen sprechen für sich: Fast $64 \%$ der untersuchten Bewohner wiesen eine mittlere bis schwere Niereninsuffizienz auf. „Das ist nicht ungewöhnlich“, erklärt PD Dr. med. Guido Schmiemann vom Institut für Public Health und Pflegeforschung an der Universität Bremen. Es zeige aber, dass bei ihnen regelmäßig der Kreatininwert bestimmt werden sollte. Nur so lasse sich beurteilen, ob eine Dosisanpassung verabreichter Medikamente erforderlich sei. „Fast 90\% der Heimbewohner leiden an Mehrfacherkrankungen und nehmen im Schnitt 6,3 Medikamente dauerhaft ein - hinzu kommt die Bedarfsmedikation. Da ist ein häufiges Abgleichen von Diagnosen und Therapieplan unerlässlich", so der Mediziner.

Zur Unterstützung von Haus- und Fachärzten sowie Pflegekräften haben die am IMREN-Projekt beteiligten Forscher Empfehlungen aus der Studie abgeleitet: So ist nun dokumentiert, welche Medikamente und Wirkstoffe in Verbindung mit einer Niereninsuffizienz häufig nicht adäquat dosiert werden und was Betroffene überhaupt nicht einnehmen sollten.

www.uni-oldenburg.de/versorgungsforschung
Gefährlicher Trend: Diabetespatientinnen im Schlankheitswahn

Mädchen und junge Frauen mit Typ-1-Diabetes haben Forschern zufolge ein besonders hohes Risiko, an einer Essstörung zu erkranken. Damit steigt auch das Risiko für diabetesbezogene Komplikationen. Über die Erkrankung am Typ-1-Diabetes wird den Patienten ein Instrument an die Hand gegeben, ihr Körpergewicht bei Bedarf zu regulieren: So lässt sich über die absichtliche Unterdosierung von Insulin bewusst eine Hyperglykämie und infolgedessen eine Ausschwemmung von Glukose über den Urin herbeiführen. Entsprechende Praktiken scheinen sich zu einem besorgniserregenden Trend vor allem unter jungen Frauen mit Typ-1-Diabetes zu entwickeln, berichten Forscher der University of Toronto.

Colton PA et al. Diabetes Care 2015; 38: 1212 1217

\section{Keimgefahr bei Muttermilchspenden}

Seit einiger Zeit kursieren Meldungen über Verunreinigungen und Panschereien bei Online-Angeboten von Muttermilch. Jeder zehnten Probe sei Kuhmilch beigemischt, so eine US-Studie. Experten weisen zudem auf die große Gefahr bakterieller oder viraler Verunreinigungen hin. Die Forderung: Milchspenden sollten den gleichen strengen Hygienevorschriften unterliegen wie Blutspenden. Die Experten raten $\mathrm{zu}$ industriell hergestellter Säuglingsmilch.

www.aerztezeitung.de

\section{Kolorektales Karzinom: Rückgang der Mortalität}

Die Mortalität beim kolorektalen Karzinom sank zwischen 1989 und 2011 in Deutschland, Österreich, der Schweiz, Großbritannien, Belgien, Tschechien, Luxemburg und Irland um mehr als $25 \%$ bei Männern und mehr als $30 \%$ bei Frauen (in Deutschland $-36,7 \%$ bzw. -47,3\%). Die Ursachen für diese positive Entwicklung sind vielfältig: ein größeres Bewusstsein für das Krankheitsbild, eine geringere Prävalenz von Risikofaktoren (Rauchen und Alkoholkonsum), der Anstieg der Teilnehmerzahlen bei Screening-Untersuchungen und bessere Behandlungsstrategien.

BMJ 2015;351:h4970; doi: 10.1136/bmj.h4970 